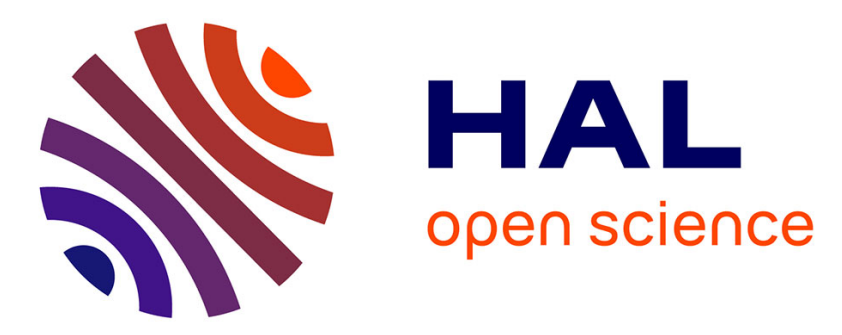

\title{
Calculs thermomécaniques pour la conception de structures réfractorisées
}

Alain Gasser, Philippe Boisse, Jacques Poirier, Yves Dutheillet

\section{To cite this version:}

Alain Gasser, Philippe Boisse, Jacques Poirier, Yves Dutheillet. Calculs thermomécaniques pour la conception de structures réfractorisées. Revue Européenne des Éléments Finis, 2002, 11 (2-4), pp.511525. 10.3166/reef.11.511-525. hal-00021132

\section{HAL Id: hal-00021132 https://hal.science/hal-00021132}

Submitted on 10 Apr 2018

HAL is a multi-disciplinary open access archive for the deposit and dissemination of scientific research documents, whether they are published or not. The documents may come from teaching and research institutions in France or abroad, or from public or private research centers.
L'archive ouverte pluridisciplinaire HAL, est destinée au dépôt et à la diffusion de documents scientifiques de niveau recherche, publiés ou non, émanant des établissements d'enseignement et de recherche français ou étrangers, des laboratoires publics ou privés. 


\title{
Calculs thermomécaniques pour la conception de structures réfractorisées
}

\author{
Alain Gasser* — Philippe Boisse* — Jacques Poirier** \\ Yves Dutheillet $* * *$
}

* Laboratoire de Mécanique des Systèmes et des Procédés, ESEM

8, rue Léonard de Vinci, F-45072 Orléans Cedex 2

\{alain.gasser,philippe.boisse\}@univ-orleans.fr

** CRDM/Sollac, Rue du Comte Jean

F-59240 Dunkerque

jacques.poirier@sollac.usinor.com

*** Electricité de France, DMA, Les renardières

F-77818 Moret-sur-Loing Cedex

Yves.Dutheillet@edf.fr

RÉSUMÉ. Les structures réfractorisées peuvent présenter des dégradations en cours de fonctionnement. Afin de limiter ces dégradations en améliorant la conception, il est nécessaire de disposer d'outils de calcul (par éléments finis) adaptés. Pour des structures simples, telles que des poches à acier, un calcul $3 D$ est réalisable. Par contre, pour des structures plus complexes, telles que des centrales à charbon, l'utilisation d'un élément simplifié est indispensable. Il s'agit ici d'un élément coque bi-couche dont le comportement est équivalent à celui de l'ensemble ossature métallique/garnissage réfractaire.

ABSTRACT. Degradations in refractorised structures can appear during using. To limit these degradations in improving the design, it is necessary to have adapted finite element computing tools. For simple structures, like steel ladles, a 3D computing can be performed. On the other hand, for more complex structures, like coal fired power plants, the use of a simplified element is essential. It is here a two-layered shell element that has an equivalent bahaviour to the set of metallic casing and refractory lining.

MOTS-CLÉS : structures réfractorisées, élément coque bi-couche équivalent, identification inverse, thermomécanique, modèle smeared crack, joints de dilatation.

KEYWORDS: refractorised structures, equivalent two-layered shell element, inverse identification, thermomechanics, smeared crack model, expansion joints. 


\section{Introduction}

De nombreuses structures contenant un fluide à haute température utilisent des garnissages réfractaires pour protéger l'ossature métallique qui ne doit pas dépasser une température maximum pour un fonctionnement correct. Les domaines d'application principaux sont ceux de la sidérurgie (Poirier, 1996) (Peruzzi et al. 1999) et de la production de l'aluminium ainsi que la production d'énergie électrique (Gordon, 1993).

Les garnissages réfractaires sont soumis à des sollicitations thermomécaniques très sévères compte tenu des niveaux de température $\left(1650^{\circ} \mathrm{C}\right.$ en sidérurgie). Les déformations résultant des gradients de température ainsi que de la différence des coefficients de dilatation thermique du réfractaire et des supports métalliques peuvent conduire à des endommagements des garnissages dont le niveau conduit, dans certains cas, à l'arrêt de l'installation. Si la conception des garnissages est basée sur une grande expérience des industries concernées (une assez bonne maîtrise des phénomènes de corrosion), il s'avère de plus en plus prometteur de développer des méthodes de simulation du comportement des structures réfractorisées sous sollicitations thermomécaniques.

Le but de cette étude est de développer des outils de calcul par éléments finis qui permettront d'apporter une aide à la conception de structures réfractorisées telles que des poches à acier (sidérurgie) ou des centrales à charbon (production d'énergie). Elles sont constituées d'une enveloppe métallique protégée du contenu chaud par un garnissage réfractaire. Ces garnissages sont constitués soit de béton réfractaire, ancré (ou non) sur le support métallique de la structure (appelé « casing »), soit de briques. Le casing inclut fréquemment des tubes pour la circulation d'eau pressurisée. Des joints de dilatation sont présents dans le réfractaire. Quatre échelles peuvent être définies dans ce problème (figure 1) : la première est celle du matériau, la seconde est locale (ancrages, tubes, joints, ...), la troisième est celle du panneau comprenant l'ensemble des composants (échelle méso) et la dernière est celle de la structure (échelle de la structure). Pour calculer une structure complète (plusieurs mètres), il est parfois possible d'effectuer un calcul 3D assez correctement (cas des poches à acier). Dans des cas de géométrie plus complexe (surtout localement), il n'est pas possible de décrire chaque détail tel que les ancres, les tubes ou les joints. La solution proposée dans ce travail est d'utiliser un élément simplifié, au comportement équivalent à celui de la paroi réfractorisée, qui sera ici un élément coque bicouche, afin de diminuer la taille du modèle éléments finis.

Cet élément coque est défini à l'échelle méso. La première couche est élastique orthotrope et modélise le casing, la seconde est élastique endommageable et représente le garnissage réfractaire ancré. Le comportement de cet élément doit être équivalent à celui de l'ensemble casing, garnissage et ancres. L'identification des paramètres de cette coque est réalisée en utilisant des essais de traction, flexion, cisaillement, ainsi que des essais thermiques, sur une cellule représentative comprenant plusieurs ancres. Les résultats de ces essais sont comparés aux 
simulations réalisées à l'aide du modèle coque. Ceci permet, grâce à une méthode inverse, d'identifier les paramètres de comportement de cette coque. Ces essais étant difficilement réalisables expérimentalement, ils ont été simulés à l'aide d'un modèle défini à l'échelle inférieure (celle des composants élémentaires). A cette échelle, le comportement du réfractaire est supposé élasto-plastique en compression et élastique-endommageable en traction, avec adoucissement après la partie élastique (modèle " smeared crack »). Ce comportement en traction est identifié à l'aide d'un essai de flexion quatre points, lié à une méthode inverse.
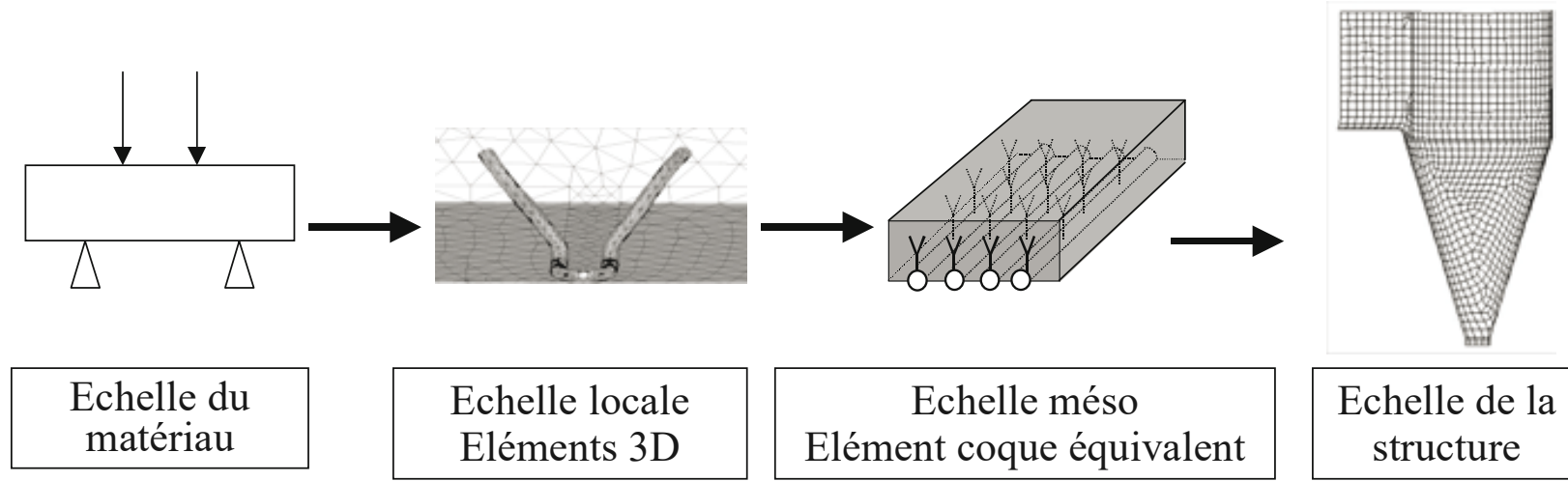

matériau

Eléments 3D

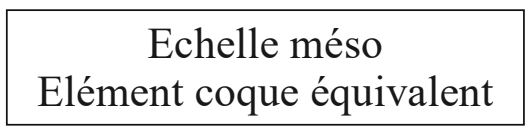
Echelle de la structure

Figure 1. Les différentes échelles du problème

Une validation expérimentale de l'approche décrite ci-dessus est présentée. Elle a été réalisée grâce à un essai de flexion quatre points. Cette approche est ensuite appliquée au calcul de structures réfractorisées complètes (un exemple est présenté dans le cas d'un élément de centrale thermique).

Un autre point important lors du calcul d'une structure réfractorisée est la prise en compte des joints de dilatation. En effet, ces joints permettent de limiter les contraintes d'origine thermique en laissant le réfractaire se dilater librement au début du chargement. Une première modélisation de ces joints est présentée dans deux cas. Le premier concerne des joints présents entre des panneaux réfractaires. Ils sont directement pris en compte dans l'élément coque bicouche équivalent par modification du modèle (qui tient compte du fait que les joints peuvent être soit ouverts, soit fermés) et adaptation des essais d'identification (les panneaux testés doivent contenir des joints). Le second cas concerne des garnissages réfractaires composés de briques. Grâce à l'identification du comportement des interfaces entre les briques, il est possible de simuler le comportement d'une cellule élémentaire d'une maçonnerie, et d'en déduire le comportement d'un matériau équivalent qui pourra servir lors des simulations. 


\section{Modélisation à l'échelle locale}

\subsection{Description du modèle}

L'échelle locale est celle des composants (tubes, ancres, joints...). Les structures étudiées sont composées d'acier et de réfractaire. L'acier a un comportement élastoplastique, tandis que le réfractaire est supposé élasto-plastique en compression et élastique-endommageable en traction, avec adoucissement (Petersson, 1985) (Cotterell et al., 1996) après la partie élastique (figure 2a). Ce dernier comportement est pris en compte à l'aide d'un modèle à fissuration diffuse («smeared crack model ») dont il existe plusieurs types (Weihe et al., 1998) :

- modèle avec une fissure fixe (Rashid, 1968): la fissure apparaît perpendiculairement à la contrainte principale maximale en mode I, et son orientation reste fixe pour un chargement plus élevé et de direction différente,

- modèle avec une fissure pivotante (Cope et al., 1980) : la fissure apparaît également perpendiculairement à la contrainte principale maximale en mode I, mais sa direction peut changer pour rester perpendiculaire à la contrainte principale maximale,

- le modèle à fissures fixes multiples (Litton, 1976) (De Borst et al., 1985) (Hibbitt et al., 1997) est identique au modèle avec une fissure fixe, mais des fissures secondaires peuvent apparaître perpendiculairement aux fissures existantes.

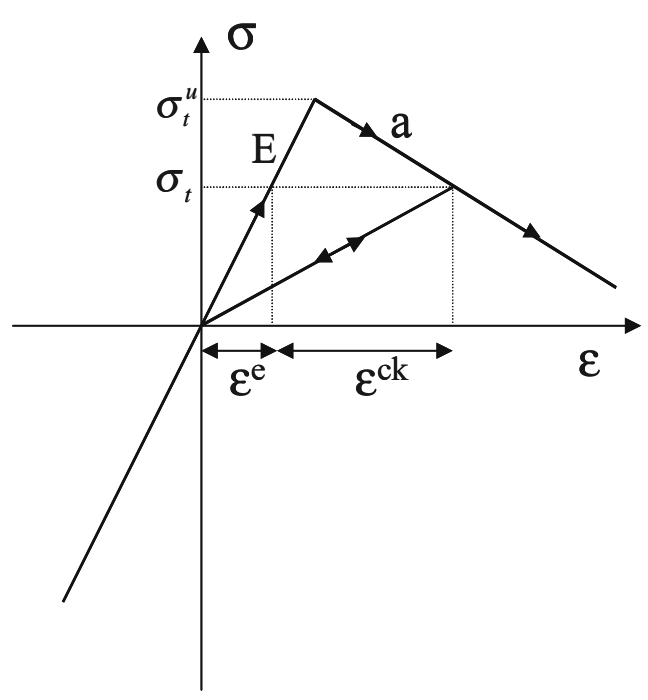

a)

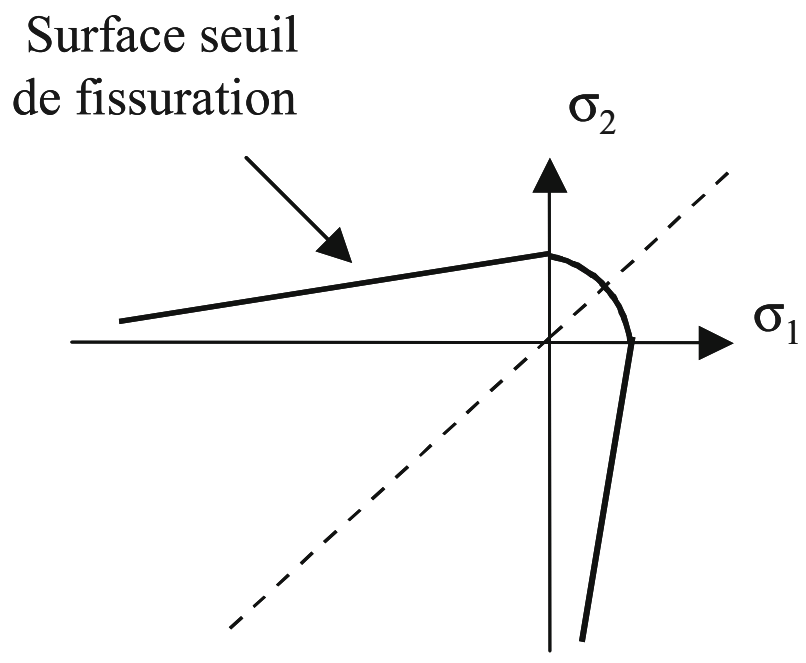

b)

Figure 2. a) Comportement uniaxial en traction/compression des réfractaires ;

b) Surface seuil de début de fissuration

C'est ce dernier modèle qui a été choisi ici. Par simplification, la partie adoucissante de la courbe de traction (figure 2a) est prise comme étant une droite de 
pente $a$. En 3D, la fissuration est détectée à l'aide d'une surface donnée par la figure $2 \mathrm{~b}$ et l'équation (1) :

$$
f=q-\left(3-b \frac{\sigma_{t}}{\sigma_{t}^{u}}\right) p-\left(2-\frac{b}{3} \frac{\sigma_{t}}{\sigma_{t}^{u}}\right) \sigma_{t}=0
$$

avec :

$$
\begin{aligned}
& p=-\frac{1}{3} \operatorname{trace}(\boldsymbol{\sigma}) \\
& q=\sqrt{\frac{3}{2} \mathbf{S}: \mathbf{S}} \\
& \mathbf{S}=\sigma+p \mathbf{I}
\end{aligned}
$$

où $\sigma_{t}^{u}$ est la contrainte de première fissuration en traction uniaxiale, $p$, la pression hydrostatique, $q$, la contrainte équivalente de Von Mises, $b$, une constante, et $\sigma_{t}$, la contrainte maximale qui peut être atteinte en traction uniaxiale après fissuration.

Du fait de la difficulté de réaliser des essais de traction sur des réfractaires de type béton, le comportement en traction (module d'Young $E$, contrainte de première fissuration $\sigma_{t}^{u}$, pente d'adoucissement $a$ ) est identifié à l'aide d'un essai de flexion quatre points (Lemaistre, 1998) relié à une méthode inverse (Marquardt, 1963) (Schnur et al., 1992). En effet, un essai de flexion est un essai de structure avec traction et compression. Ainsi une identification directe n'est pas possible.

\subsection{Validation}

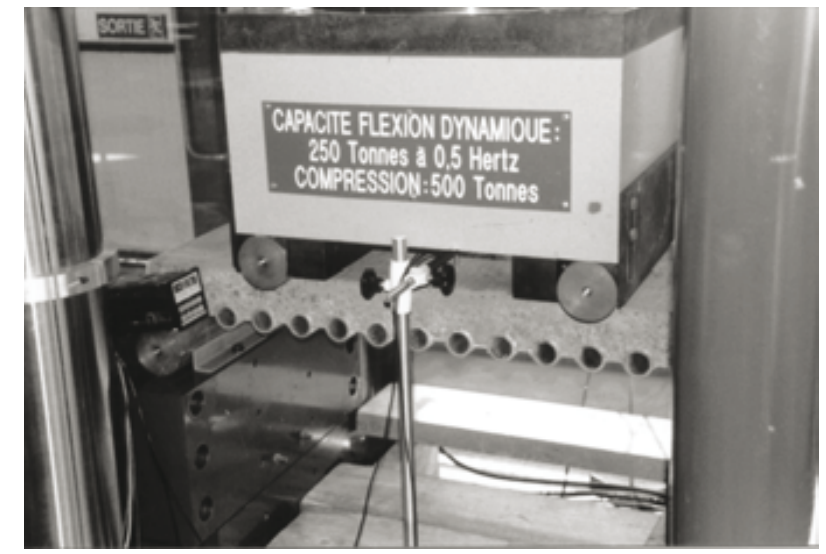

a)

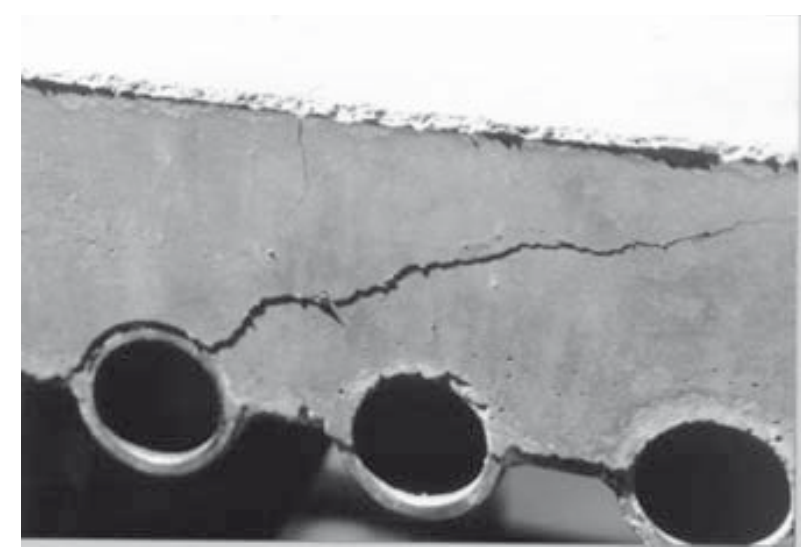

b)

Figure 3. Essai de flexion 4 points sur panneau : a) essai, b) fissuration 
Un essai de flexion quatre points a été réalisé sur un panneau réfractorisé $(1,2 \mathrm{~m}$ de long) contenant plusieurs tubes et ancres (figure 3). La simulation de cet essai (figure 4) est comparée aux résultats expérimentaux. Les fissures apparaissent aux sommets des tubes (figures $3 b$ et $4 b$ ), et les courbes effort/déplacement sont en bonnes cohérence (figure 5).

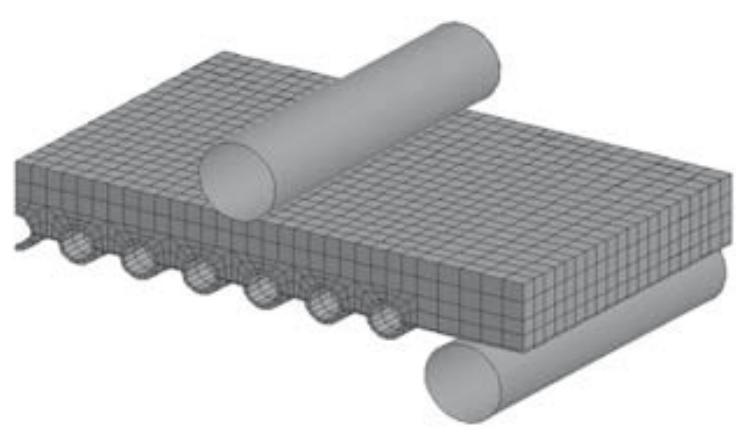

a)

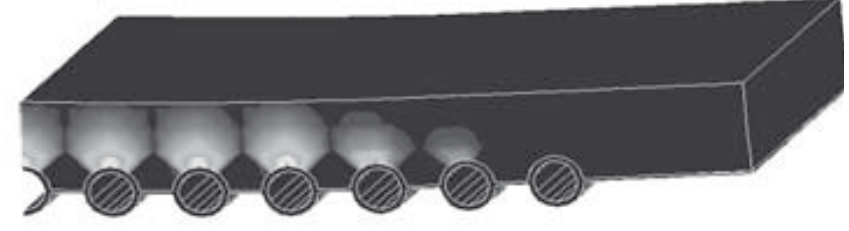

b)

Figure 4. Simulation de l'essai de flexion sur panneau : a) maillage de la moitié de la structure, b) endommagement

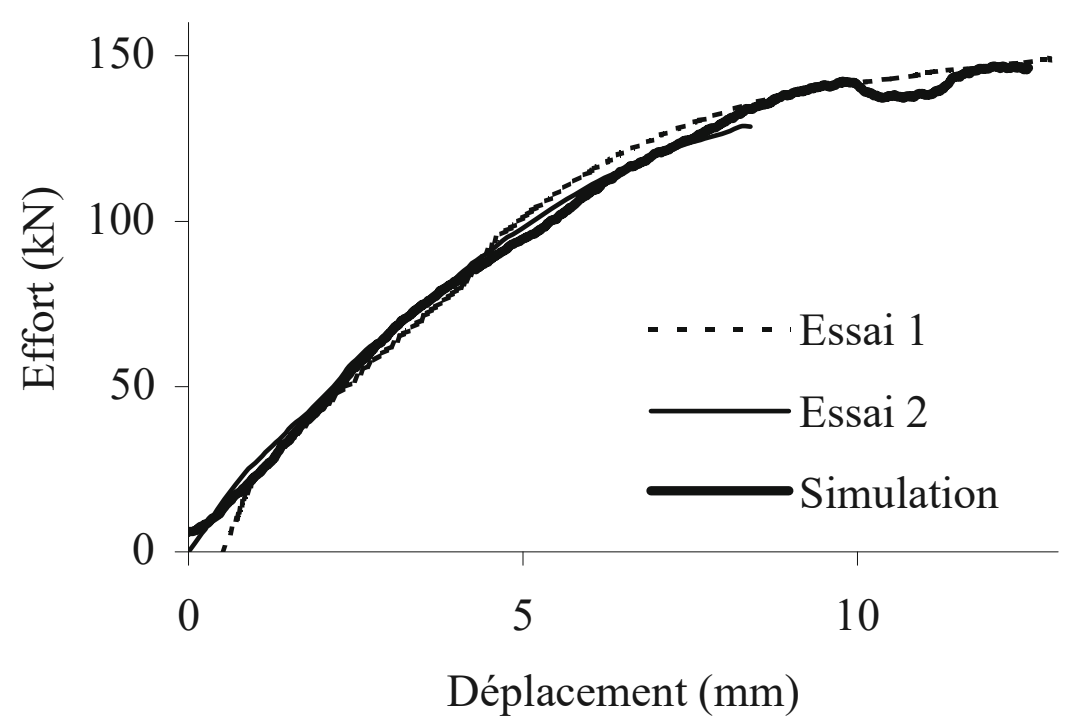

Figure 5. Flexion sur panneau : comparaison essais/simulation pour les courbes effort/déplacement

\subsection{Exemple de simulation $3 D$ : une poche à acier}

Le modèle 3D présenté ci-dessus peut être utilisé pour calculer certaines structures qui ne sont pas trop complexes, telles des poches à acier (Derré et al., 2000) (qui ne comportent ni ancres, ni tubes de refroidissement). La figure 6a montre le maillage du quart d'une telle poche comprenant deux couches de 
réfractaires : une couche d'usure (en briques) et une couche de sécurité (fabriqué à l'aide de béton réfractaire). En première approximation, la couche d'usure a été modélisée par un volume monobloc homogène.

La difficulté du calcul est principalement liée à une bonne définition des sollicitations thermiques qui sont très sévères (cycle de remplissage de la poche en acier liquide à $1650^{\circ} \mathrm{C}$, rayonnement, convection, ...), à une bonne prise en compte du comportement des matériaux (déshydratation, évolution des caractéristiques avec la température, endommagement (Berthaud et al., 1998), plasticité) et à une bonne gestion de la refermeture des joints (contact, frottements et thermomécanique couplée). Des contraintes importantes apparaissent en tôlerie (figure 6b) et dans les réfractaires, provoquant la fissuration de la face « froide » de la couche de sécurité (figure 6c).

Cette modélisation permet de dimensionner la tôlerie et les épaisseurs de réfractaires afin de ne pas dépasser les limites élastiques ou à rupture des différents matériaux.

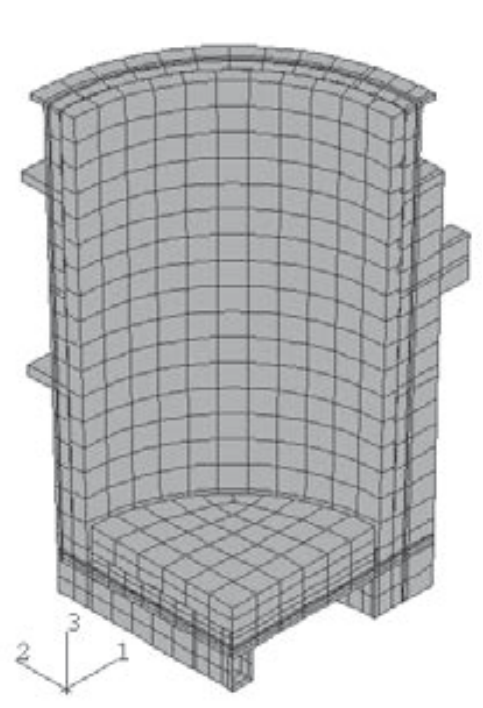

a)

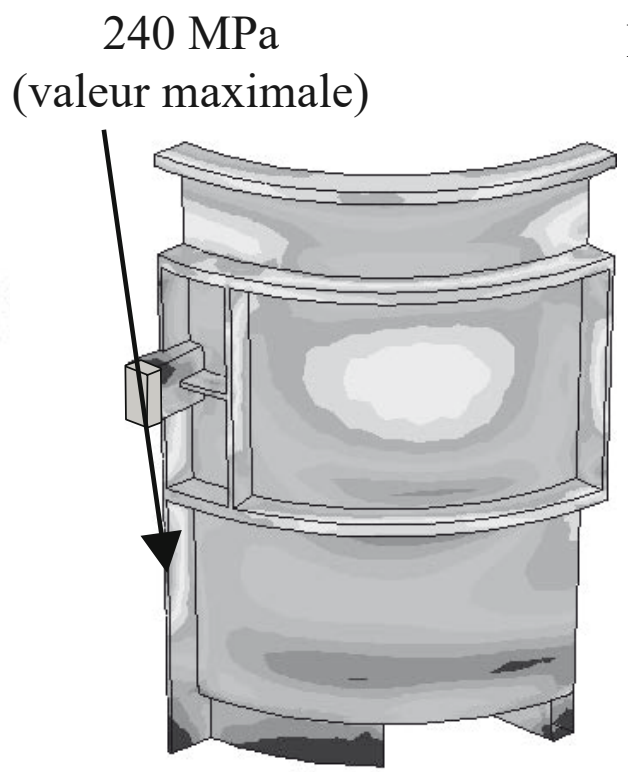

b)
Endommagement maximum

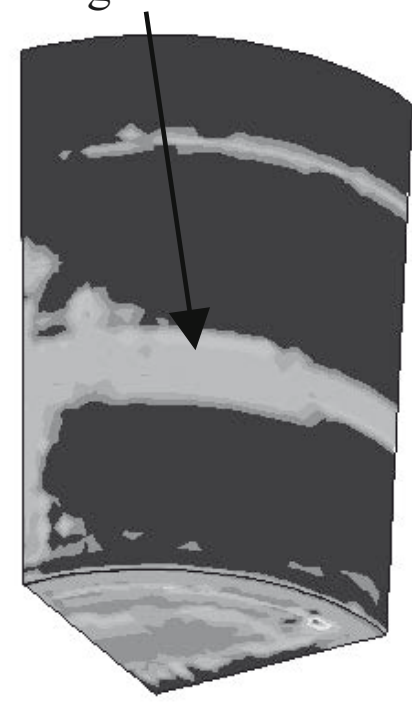

c)

Figure 6. Calcul d'une poche à acier : a) maillage d'un quart de poche, b) contraintes de Von Mises en tôlerie, c) endommagement de la face "froide » de la couche de sécurité

\section{Modélisation à l'échelle locale}

\subsection{Elément coque bi-couche}

Dans certains cas, la structure est trop complexe pour réaliser une modélisation 3D. La solution proposée est de définir un élément simplifié, à l'échelle méso, qui est une coque comportant deux couches (figure 7) : 
- la première (représentant le casing tubé dans une direction) est élastiqueorthotrope, avec 9 coefficients (caractéristiques mécaniques $E_{1}, E_{2}, v_{12}, G_{12}, G_{13}, G_{23}$, coefficient de dilatation thermique $\alpha$, conductivité $\lambda$, chaleur spécifique $C_{p}$ ),

- la seconde (représentant le garnissage réfractaire ancré) est élastiqueendommageable, avec 7 coefficients $\left(E, v\right.$, contrainte de première fissuration $\sigma_{t}^{u}$, pente d'adoucissement $a, \alpha, \lambda, C_{p}$ ). Le modèle d'endommagement est le même que celui présenté pour le modèle à l'échelle locale.

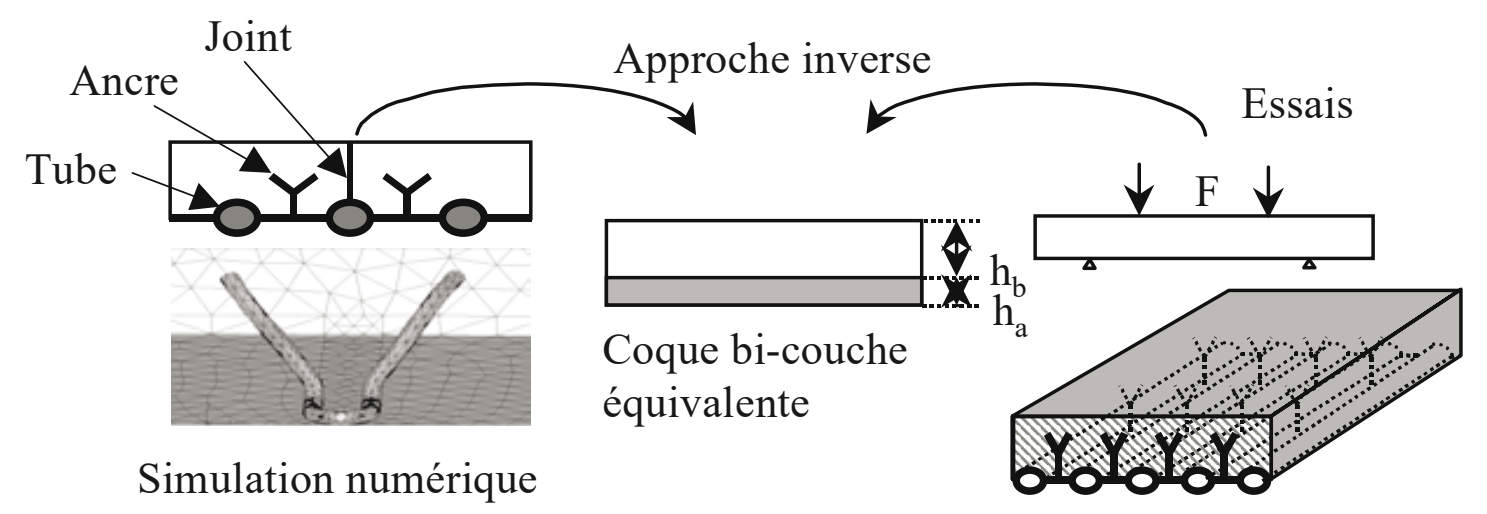

Figure 7. Modélisation d'une cellule 3D (garnissage, ancres et casing tubé) par un élément coque bi-couche équivalent, et identification par approche inverse

A ces 16 paramètres il faut ajouter les épaisseurs $h_{1}$ et $h_{2}$ de chaque couche. Le comportement de cet élément doit être équivalent à l'ensemble casing tubé, ancres et garnissage. Il est à noter que dans ce paragraphe les joints de dilatation ne sont pas pris en compte.

\subsection{Identification}

L'identification des 18 paramètres de cette coque est réalisée en utilisant des essais de traction, flexion, cisaillement et de thermique, sur une cellule représentative contenant plusieurs ancres. Les résultats de ces essais sont comparés aux simulations obtenues à l'aide du modèle coque. Ceci permet, par utilisation d'une méthode inverse (Marquardt, 1963) (Schnur et al., 1992), d'identifier les paramètres de la coque bi-couche. Mais ces essais étant difficilement réalisables expérimentalement (au vu de la taille des panneaux), ils ont été simulés à l'aide du modèle 3D présenté dans le paragraphe précédent (Boisse et al., 2001) (à l'échelle locale des composants).

L'identification est réalisée en trois étapes. La première consiste à déterminer les huit coefficients mécaniques élastiques et les deux épaisseurs à l'aide de deux essais de traction (sens longitudinal et transversal), deux essais de flexion quatre points (sens longitudinal et transversal) et un essai de cisaillement dans le plan (figure 8). 
La deuxième étape permet de déterminer les deux paramètres d'endommagement de la couche élastique-endommageable de la coque grâce à deux essais de traction (sens longitudinal et transversal).

Tractions $\rightarrow$ E, $v, E_{1}, E_{2}, v_{12}$

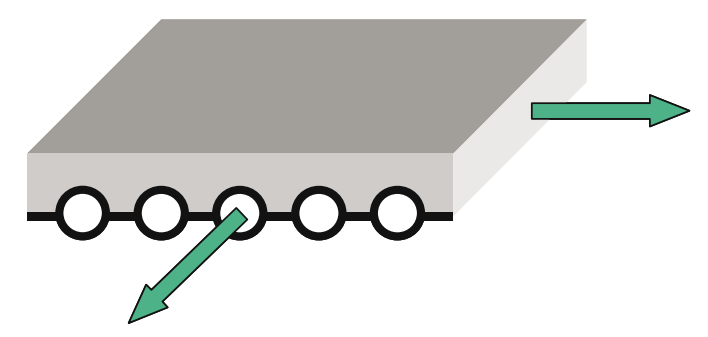

Cisaillement $\rightarrow \mathrm{G}_{12}$

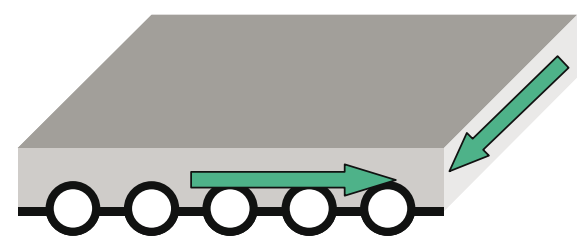

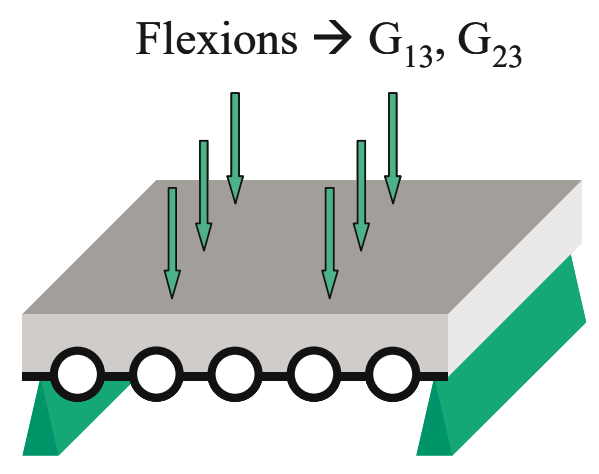

$\mathrm{h}_{\mathrm{a}}, \mathrm{h}_{\mathrm{b}}$

Pression surfacique

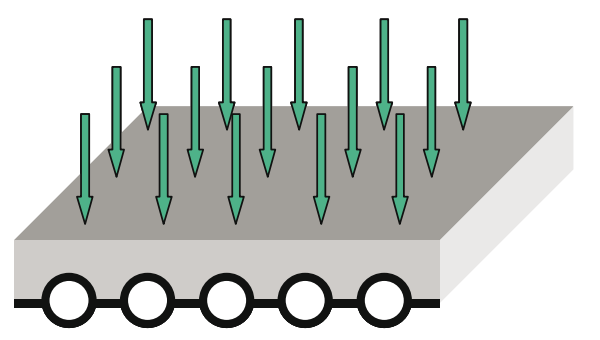

Validation

Figure 8. Essais nécessaires à l'identification et la validation des paramètres mécaniques élastiques de la coque bi-couche équivalente

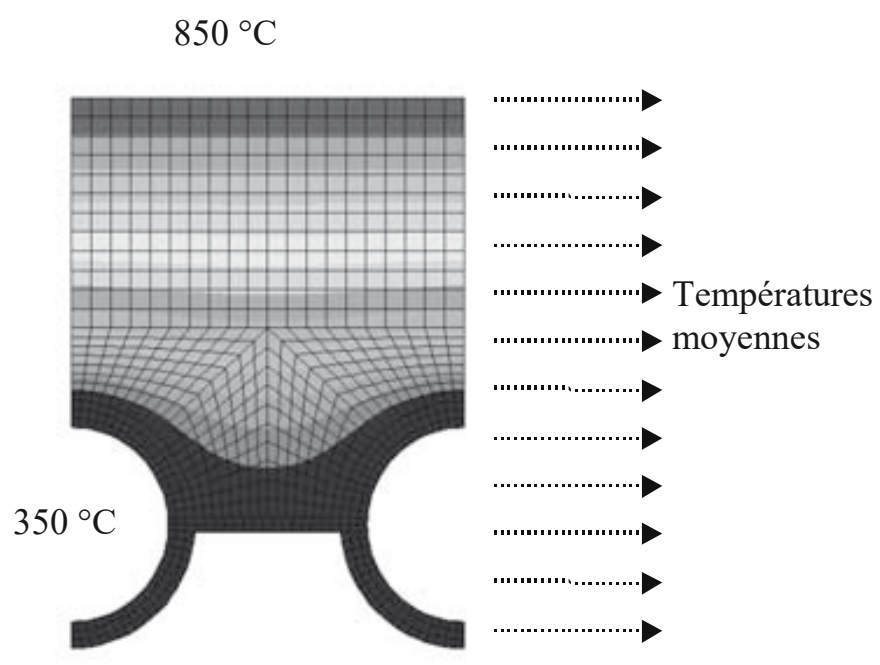

Figure 9. Simulation d'un essai de thermique $3 D$ 
Enfin, la troisième étape permet l'obtention des six paramètres thermiques et thermomécaniques de la coque à l'aide d'un essai de thermique : une cellule 3D élémentaire est soumise à une température de $350^{\circ} \mathrm{C}$ à l'intérieur des tubes et $850^{\circ} \mathrm{C}$ sur la face extérieure (figure 9). La difficulté se situe dans la comparaison des températures entre les modèles 3D et coque. En effet, la température n'est pas constante sur une horizontale du modèle 3D (figure 9). Pour cette raison, les comparaisons se font par rapport à la moyenne des températures sur une ligne donnée du modèle 3D (figure 9).

\subsection{Validation}

L'approche précédente est validée à l'aide d'un essai qui n'a pas été utilisé pour l'identification des paramètres. Il s'agit d'un panneau encastré sur ses quatre bords, soumis à une pression uniforme (figure 8). La comparaison des déplacements (figure 10) entre les simulations 3D et coque montre que les différences sont inférieures à $8 \%$. La différence importante se situe au niveau du temps de calcul car le modèle 3D comporte 20000 degrés de liberté contre seulement 700 au modèle coque bi-couche.

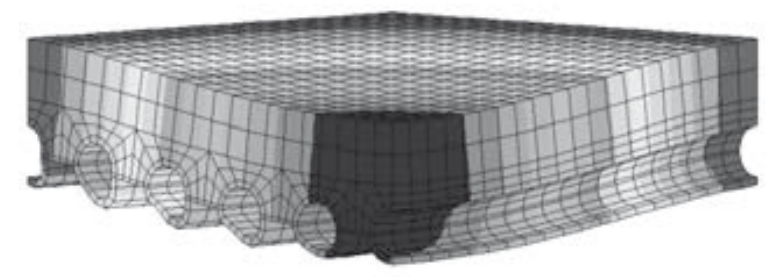

(a)

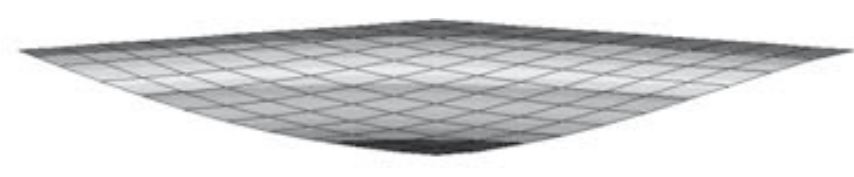

(b)

Figure 10. Comparaison des déplacements d'un quart de panneau encastré soumis à une pression, modélisé en $3 D(a)$ et en coque (b)

\subsection{Exemple de calcul d'une structure : un cyclone}

Cet élément coque bicouche est ensuite appliqué à la simulation d'une structure complète, par exemple un cyclone (partie d'une centrale à charbon à Lit Fluidisé Circulant, figure 11a). L'endommagement de la couche de réfractaire dû au chargement thermique du cyclone $\left(850^{\circ} \mathrm{C}\right.$ sur la partie interne, $350^{\circ} \mathrm{C}$ sur la partie externe) est présentée figure 11. Cet endommagement est important parce que les joints de dilatation, qui permettent de baisser les contraintes, n'ont pas été pris en compte. Il est donc nécessaire de les introduire dans le modèle. 


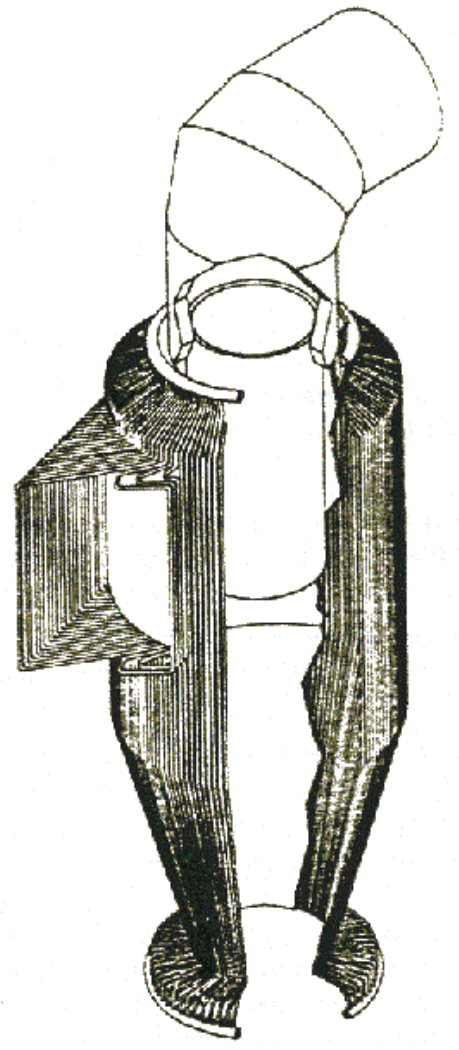

a)

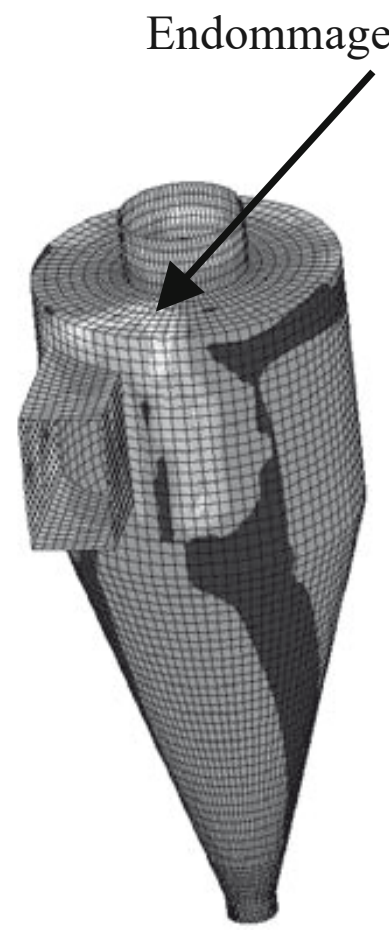

b)

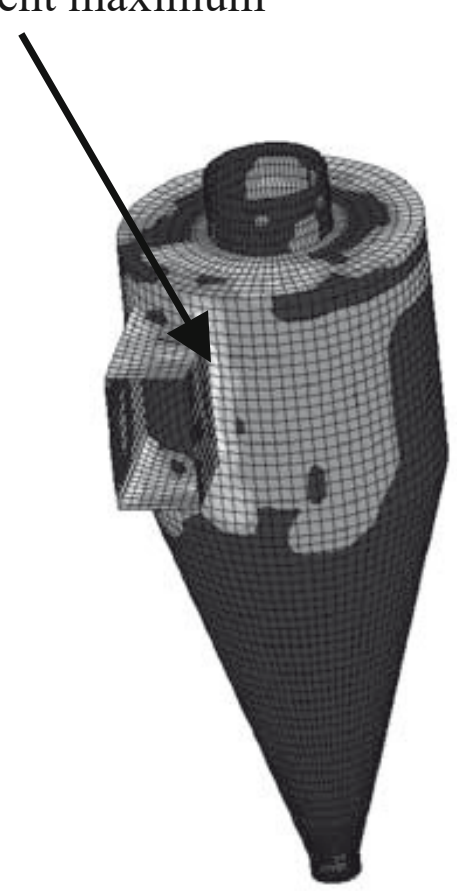

c)

Figure 11. Calcul d'un cyclone a) avec des éléments coques bi-couches. Endommagement de la couche de réfractaire : b) face interne, c) face externe

\subsection{Joints de dilatation}

\subsubsection{Cas de l'élément coque bicouche}

Afin de prendre en compte les joints de dilatation dans le cas d'un cyclone, la même approche que dans le paragraphe 3.1 est utilisée, mais les résultats $3 \mathrm{D}$ sont obtenus à partir de panneaux réfractorisés comportant deux joints perpendiculaires (figure 12a). La simulation d'essais de traction/compression sur de tels panneaux montre que la courbe contrainte/déformation présente deux pentes distinctes (figure $12 \mathrm{~b})$ : la première $\left(\mathrm{E}_{\mathrm{t}}\right)$ correspond à des joints ouverts, la seconde $\left(\mathrm{E}_{\mathrm{c}}\right)$ correspondant à des joints fermés. Une solution est de prendre deux fois plus de paramètres mécaniques pour la coque bi-couche : un jeu de paramètres pour des joints ouverts, et un jeu pour des joints fermés.

En ce qui concerne les paramètres thermiques, on considère qu'ils sont identiques joints fermés ou ouverts, car le flux thermique est essentiellement perpendiculaire aux parois, donc peu perturbé par les joints. 


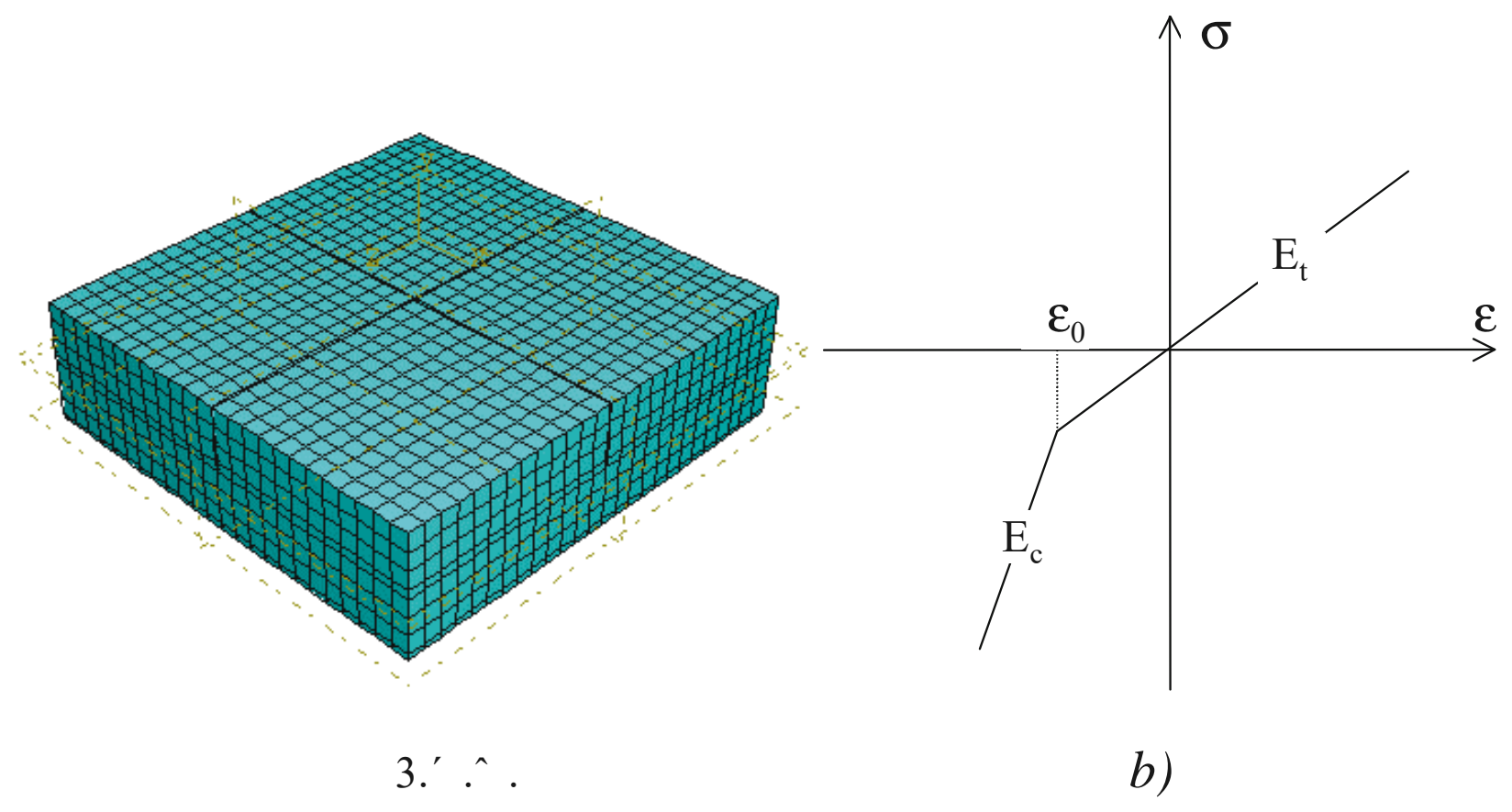

Figure 12. a) Maillage du volume élémentaire représentatif avec deux joints de dilatation perpendiculaires; b) Comportement en traction-compression de ce volume

\subsubsection{Modélisation d'un garnissage réfractaire composé de briques}

Dans le cas de nombreuses applications, une couche de réfractaire n'est pas monolithique, mais composée de briques (comme pour la couche d'usure de la poche à acier, § 2.3). Du fait des défauts de surface entre les briques, il persiste un espace (joint) entre ces briques, ce qui réduit les contraintes d'origine thermique en début de chargement. L'idée est alors de trouver le comportement équivalent d'un mur de briques afin de continuer à modéliser la couche d'usure par un matériau homogène (la modélisation de chaque brique n'étant pas possible). Ce comportement équivalent est à nouveau identifié par méthode inverse à partir d'essais de traction/compression et cisaillement sur un ensemble de briques représentatif (figure 14a). Au vu de la difficulté de réaliser de tels essais, ils sont simulés à l'aide d'un modèle à l'échelle inférieure. A cette échelle, il est nécessaire d'identifier le comportement d'un joint entre deux briques. Ce joint est modélisé par un contact avec :

- un comportement tangentiel : modèle de frottement de Coulomb,

- un comportement normal : la pression de contact évolue en fonction de la distance entre les deux briques.

Deux essais sont nécessaires pour identifier le comportement de ce contact :

- un essai de frottement : on superpose deux briques sur un plan incliné. L'angle de début de glissement donne le coefficient de frottement, 
- un essai de compression sur deux briques. La courbe obtenue (figure 13a) présente deux zones: la première, non linéaire, représente l'écrasement des rugosités à l'interface ; la deuxième, linéaire, représente la rigidité des briques.

Ce dernier essai permet de déterminer l'espacement initial entre deux briques (c'est l'intersection du prolongement de la partie linéaire avec l'axe des abscisses), ainsi que l'évolution de la pression de contact entre les deux briques (figure 13b) identifiée par méthode inverse.

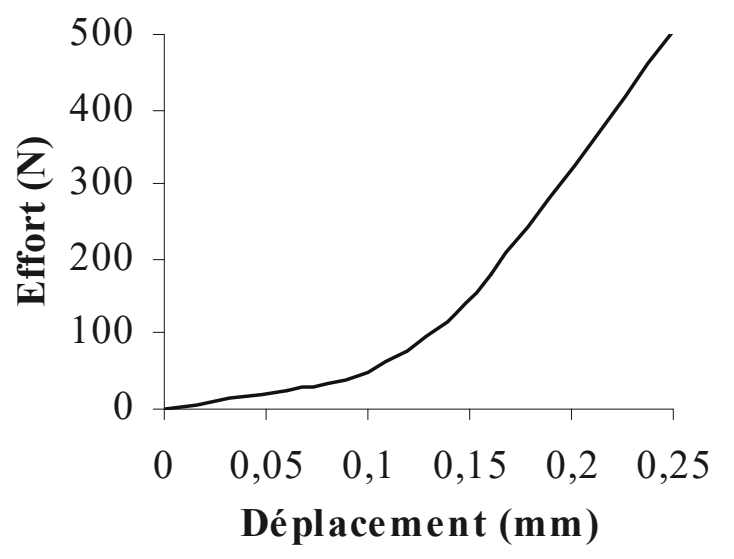

a)

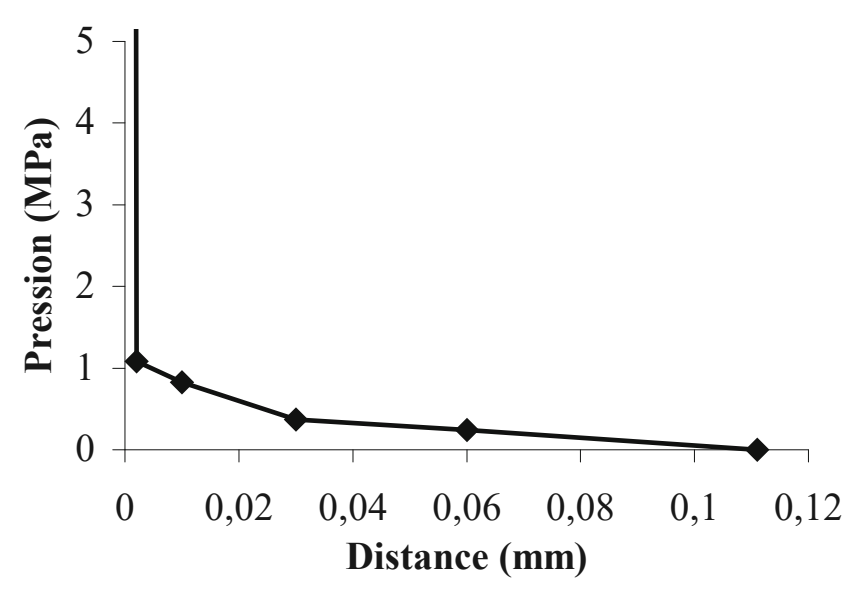

b)

Figure 13. a) Courbe de compression de deux briques, b) Courbe de comportement normal du joint

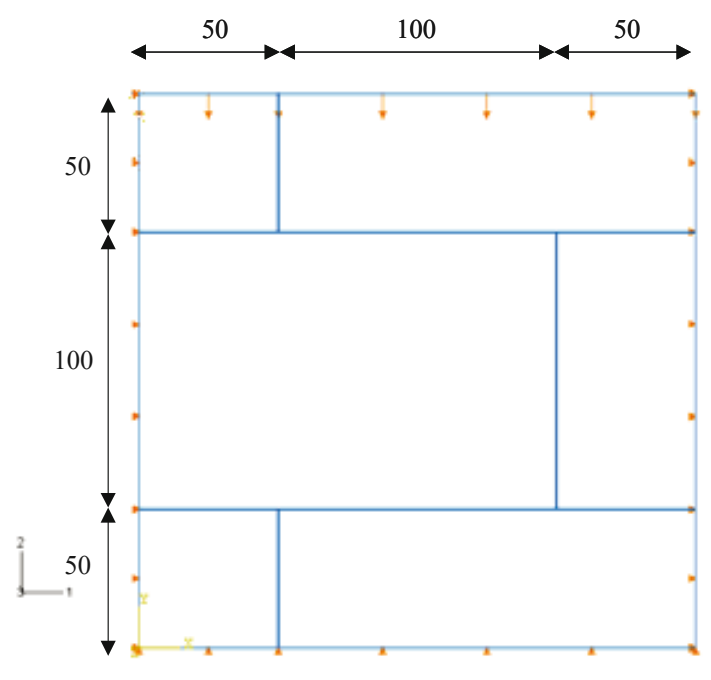

a)

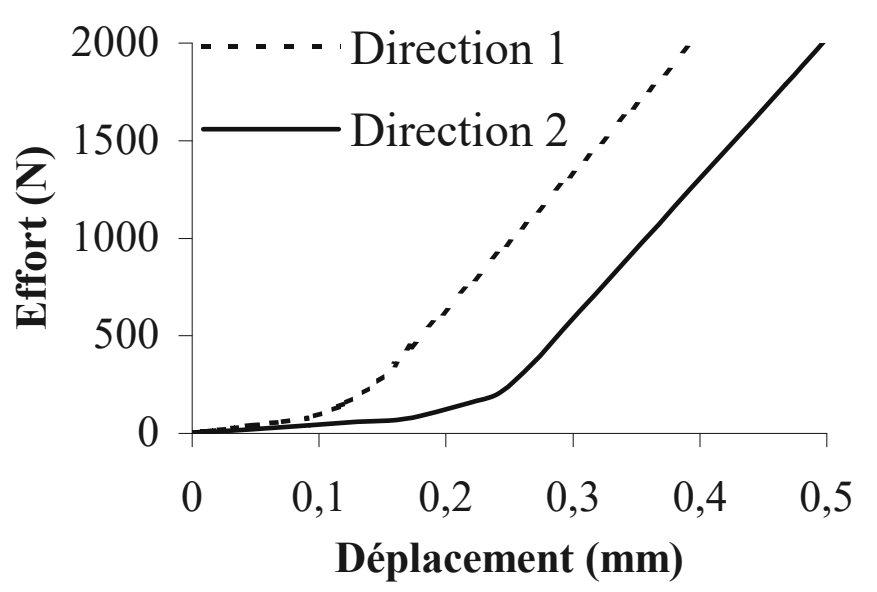

b)

Figure 14. a) Cellule élémentaire d'un mur de briques soumise à de la compression dans la direction 2 ; b) Courbes de comportement en compression dans les deux directions principales 
Ce modèle permet de simuler des essais de traction/compression et de cisaillement sur une cellule élémentaire d'un mur (figure 14a). La figure 14b donne par exemple le comportement en compression dans les deux directions principales.

La dernière étape, en cours, consiste à définir le modèle équivalent et de l'identifier par méthode inverse.

\section{Conclusion}

Afin de simuler par éléments finis une structure réfractorisée comportant des centaines d'ancres et de tubes, il est nécessaire d'utiliser un élément simplifié. L'élément proposé dans cette étude est une coque composite à deux couches. Les paramètres thermiques et mécaniques sont identifiés à l'aide d'une méthode inverse reliée à différents essais $3 \mathrm{D}$. Du fait de la difficulté à réaliser ces essais expérimentalement, ils ont été simulés à l'aide d'un modèle 3D développé à l'échelle locale (celle des composants). Cette approche a été validée par un essai de flexion quatre points sur un panneau réfractorisé.

Le modèle 3D à l'échelle locale permet, par exemple, de simuler la fissuration du réfractaire dans une poche à acier, alors que l'élément coque bi-couche permet de simuler des structures plus complexes telles qu'un cyclone. Cette dernière simulation montre qu'il est indispensable de prendre en compte les joints de dilatation présents dans les réfractaires afin de diminuer les contraintes d'origine thermique.

Une première modélisation de ces joints a été présentée, aussi bien dans le cas de l'élément simplifié (où deux fois plus de paramètres mécaniques sont nécessaires), que dans le cas d'une structure contenant des briques réfractaires.

L'approche présentée est un outil qui permet d'estimer l'endommagement de structures réfractorisées soumises à des chargements thermomécaniques, et ainsi apporter une aide à la conception de ces structures afin de limiter la fissuration.

\section{Remerciements}

Les auteurs tiennent à remercier les sociétés EDF et Sollac/Usinor pour leur soutien dans cette étude.

\section{Bibliographie}

Berthaud Y., Robin J. M., Schmitt N., Poirier J., Themines D., Thermomechanical behaviour of magnesia carbon refractory ceramics, British Ceramic Transactions, vol. 97, $\mathrm{n}^{\circ} 1$, 1998, p. 1-10. 
Boisse P., Gasser A., Poirier J., Rousseau J., Simulations of thermomechanical behavior of composite refractory linings, Composites Part B: Engineering, vol. 32, $\mathrm{n}^{\circ}$ 5, 2001, p. 461-474.

Cope R. J., Rao P. V., Clark L. A., Norris P., Modelling of reinforced concrete behaviour for finite element analyses of bridge slabs, Numerical Methods for Nonlinear Problems I, vol. al. (Eds), p. 457-470.

Cotterell B., Mai Y. W., Fracture mechanics of cementitious materials, Blackie Academic \& Professional, 1996.

De Borst R., Nauta P., Non-Orthogonal Cracks in a Smeared Finite Element Model, Engineering Computations, vol. 2, n 1985, p. 35-46.

Derré V., Gasser A., Boisse P., Poche à acier de 270 tonnes à tenue améliorée, Rapport Usinor/LMSP, Orléans (France), 2000.

Gordon E. D., Refractories in CFB applications, 12th International Conference on Fluidized Bed Combustions, San Diego (USA), 1993, p. 967-983.

Hibbitt, Karlsson, Sorensen, Theoretical Manual of Abaqus Code, HKS Inc., 1997.

Lemaistre H., Etude des propriétés thermomécaniques de divers réfractaires, Thèse de l'INSA de Lyon (France), 1998.

Litton R. W., A contribution to the analysis of concrete structures under cyclic loading, Thèse de l'University of California, 1976.

Marquardt D. W., An algorithm for least squares estimation of nonlinear parameters, J. Soc. Indus. Appl. Math., vol. 11, n² 2, 1963, p. 431-441.

Peruzzi S., Poirier J., Glandus J. C., Huger M., Numerical study of the in-serve behaviour of refractory parts used in continuous casting, 6th European Ceramic Society Conference, Brighton (UK), 1999, p. 161-162.

Petersson P. E., Crack growth developpement of fracture zones in plain concrete and similar materials, Rapport de Lund Institute of Technology, 1985.

Poirier J., Recent tendencies in refractories in relation with service in the steel industry, 39th Colloquium on Refractories, Aachen (Germany), 1996, p. 6-16.

Rashid Y. R., Ultimate strength analysis of prestressed concrete pressure vessels, Nuclear Engineering and Design, vol. 7, $\mathrm{n}^{\circ} 1968$, p. 334-344.

Schnur D. S., Zabaras N., An inverse method for determining elastic material properties and a material interface, International Journal for Numerical Methods in Engineering, vol. 33, $\mathrm{n}^{\circ}$ 1992, p. 2039-2057.

Weihe S., Kröplin B., de Borst R., Classification of smeared crack models based on material and structural properties, International Journal of Solids and Structures, vol. 35, $\mathrm{n}^{\circ} 12$, 1998, p. 1289-1308. 